\title{
Designing RBFNNs Using Prototype Selection
}

\author{
Ana Cecilia Tenorio-González, José Fco Martínez-Trinidad, \\ and Jesús Ariel Carrasco-Ochoa \\ National Institute for Astrophysics, Optics and Electronics \\ Luis Enrique Erro No. 1, Sta. María Tonanzintla, Puebla, México, \\ C.P. 72840 \\ \{catanace17, fmartine, ariel\} @ ccc.inaoep.mx
}

\begin{abstract}
Performance and accuracy of a neural network are strongly related to its design. Designing a neural network involves topology (number of neurons, number of layers, number of synapses between layers, etc.), training synapse weights, and parameter selection. Radial basis function neural networks (RBFNNs) could additionally require some other parameters, for example, the means and standard deviations if the activation function of neurons in the hidden layer is a Gaussian function. Commonly, Genetic Algorithms and Evolution Strategies have been used for automatically designing RBFNNs In this work, the use of prototype selection methods for designing a RBFNN is proposed and studied. Experimental results show the viability of designing RBFNNs using prototype selection.
\end{abstract}

Keywords: Neural networks, RBFNN, prototype selection, supervised classification.

\section{Introduction}

Supervised classification is one of the most important problems into Pattern Recognition. Given a dataset, described by a set of attributes, for which the true classlabel is known (training set). The supervised classification problem consists in designing a classifier using the training set to automatically classify unseen data. Neural networks $[17,18]$ are among the most widely used supervised classifiers.

Neural networks are typically formed by a set of parallel processing units (neurons) interconnected among them. Neurons are organized in layers depending of their function in the network (input, hidden or output). Each neuron has associated an activation (transfer) function used for combining its inputs and weights to produce an output value. Among the different types of neural networks, radial basis function neural networks (RBFNNs) have been widely used due to its reduced number of layers and its good results in supervised classification problems [2, 3, 5-8, 18]. A RBFNN is a three-layer feed-forward network that uses radial basis functions (normally a Gaussian), as transfer functions for the hidden layer neurons. A typical RBFNN is illustrated in Figure 1. The number of nodes in the input layer is determined by the number of attributes used for describing the data in the problem to solve. In the hidden layer, the processing is made using a nonlinear transfer function 
(commonly a Gaussian). In the output layer there is a neuron for each class in the problem. The neurons in the output layer perform a simple weighted sum, for combining the outputs in the hidden layer, producing a linear output. During the training, the weights between the internal and the output layers are adjusted (weights between the input and the internal layers of a RBFNN are equal to 1.0, which can be interpreted as no weights). When an unseen object is presented to the network the class associated to the neuron with the highest value in the output layer is assigned to the object.

Generally, the automatic design of a RBF neural network has been done using Genetic Algorithms and Evolution Strategies [1 -7, 14, 18]. In this paper, we present a new method for designing RBFNNs for multiclass problems, based on prototype selection. Our method designs a RBFNN reducing the number of neurons in the hidden layer without affecting the classification accuracy.

The rest of the paper is organized as follows: Section 2 presents a review of the most recent methods for designing RBFNNs. Section 3 introduces the proposed method for designing RBFNNs for multiclass problems, based on prototype selection. Section 4 shows the experimental results, and Section 5 presents our conclusions and future work.

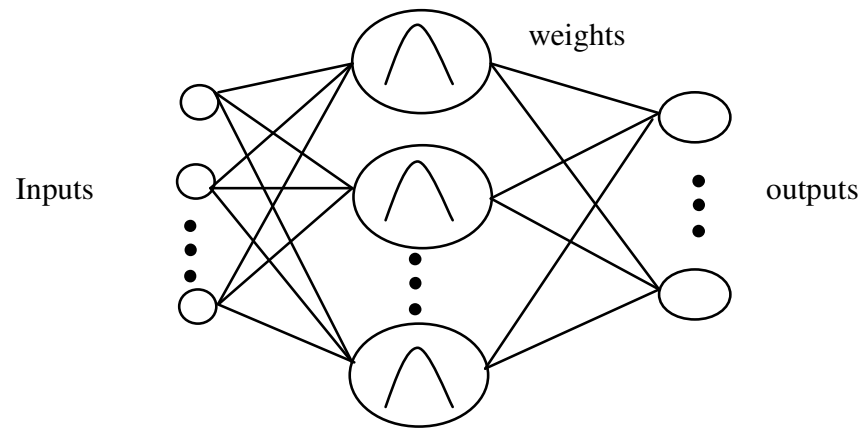

Fig. 1. RBFNN structure

\section{Related Work}

Most of the methods for automatic design of a RBFNN are based on Genetic Algorithms or Evolution Strategies. In [1], a review of evolutionary methods for designing neural networks is presented. In this work, the importance of a good design of a neural network is established; several evolutionary algorithms for modifying the network topology as well as network parameters are described and compared. Examples of evolutionary methods for automatic design of RBFNNs are [2-7]. From these, we have chosen the most recent $[5,7]$ for comparing the method proposed in this work.

In [5] a co-operative co-evolutionary algorithm (Co-CEA) is used for modifying the topology and parameters (means and standard deviations for internal neurons) of a RBFNN. First, DRSC (Decaying Radius Selection Clustering) is used to compute the 
initial hidden neurons based on the training set, and the cluster distribution is used for initializing the standard deviations. Then, the neurons of the hidden layer are clustered using a modification of the k-means algorithm. Later, each cluster is divided in overlapped subsets, which will be the individuals of a population that will evolve for each cluster. After each generation, the best individual (subset of neurons) from each cluster is selected to build the hidden layer of a RBFNN $\Theta^{*}$ (at the beginning a representative of each cluster is selected for $\Theta^{*}$ ). The fitness of an individual is based on: a) the accuracy, over a validation dataset, of the RBFNN obtained by replacing in $\Theta^{*}$ the best individual of the corresponding cluster by the evaluated individual; and, b) how many objects, misclassified by most of the RBFNNs built for all other individuals, can be correctly classified by the RBFNN of (a). Using this fitness, a roulette wheel selection is used to select individuals for the next generation; and elitist selection is used for updating $\Theta^{*}$ after each generation. After a predetermined number of iterations, the last $\Theta^{*}$ is selected as the final estimation of the RBFNN. An extension of this work was presented in [6], where the RBFNN is designed using elliptical basis functions. In [6], a co-operative co-evolutionary algorithm is also used for designing a RBFNN but, instead of using DRSC, a random initialization of the hidden layer neurons is done.

In [7], a method for designing RBFNNs for two class problems using symbiosis is presented. Symbiosis is done by means of co-evolution of two populations. For the first population, an evolutionary method is used for obtaining a set of parameters for EvRBF[14], which, for each individual of the first population, evolves a second population for designing a RBFNN. The accuracy of the best RBFNN found by EvRBF is used as fitness of the corresponding individual of the first population. Each individual of the second population $(\mathrm{EvRBF})$ is a set of neurons for the hidden layer of a RBFNN. EvRBF uses special crossover and mutation operators for evolving this population in order to modify the topology (size) and parameters (means and standard deviations) of a RBFNN. Tournament selection is done for each generation, using, as fitness, the accuracy of the RBFNN built with the neurons of an individual.

There are other methods for designing RBFNNs, which are not based on evolutionary techniques. In [9], an approach based on clustering is proposed for designing a generalized RBFNN, using clusters obtained by analyzing the input data and their expected output. In this work, the size and parameters of the network are obtained. First, the output space is clustered using $k$-means, and for each group, a fuzzy set is created. These fuzzy sets are used for clustering the input data using a contextbased fuzzy clustering algorithm. Using the clusters of the input data, a generalized RBFNN is built. In [8], fuzzy clustering is used for obtaining the means of the neurons in the hidden layer of a RBFNN; nevertheless the topology of the network is not modified.

As it can be seen, most of the methods for designing RBFNNs are based on genetic algorithms or evolution strategies. Those methods that follow a different approach have been designed for a variation of the RBFNNs or only modify the parameters of a RBFNN, but not its topology. For this reason, in this paper we compare our proposed method only against methods based on genetic algorithms and evolution strategies. 


\section{Proposed Method}

In this paper, we address the automatic design of a RBFNN through prototype selection. As we can observe in the related work, for the automatic design of a RBFNN, the majority of the works choose some instances in the training set as neurons for the hidden layer. Following this idea, for building the hidden layer, we could use one neuron for each instance in the training set; however to proceed in this way could negatively affect the network performance, since usually in the training set there are some instances which are redundant or noise. Since, the main goal of instance selection is to choose a subset of instances from the training set such that the selected subset of instances does not contain superfluous instances and, at the same time, this subset would produce as high classification accuracy as possible, we propose to use prototype selection for designing the hidden layer of a RBFNN. The hypothesis is that a RBFNN designed in this way can have similar or better results in accuracy than those results obtained by the networks designed by evolutionary approaches.

In figure 2, we show the main steps of our method, the prototype selection methods are applied to the training data in order to choose some representative instances. Then these instances are used to create the neurons in the hidden layer of the RBFNN. Finally, the network designed in this way is trained and evaluated.

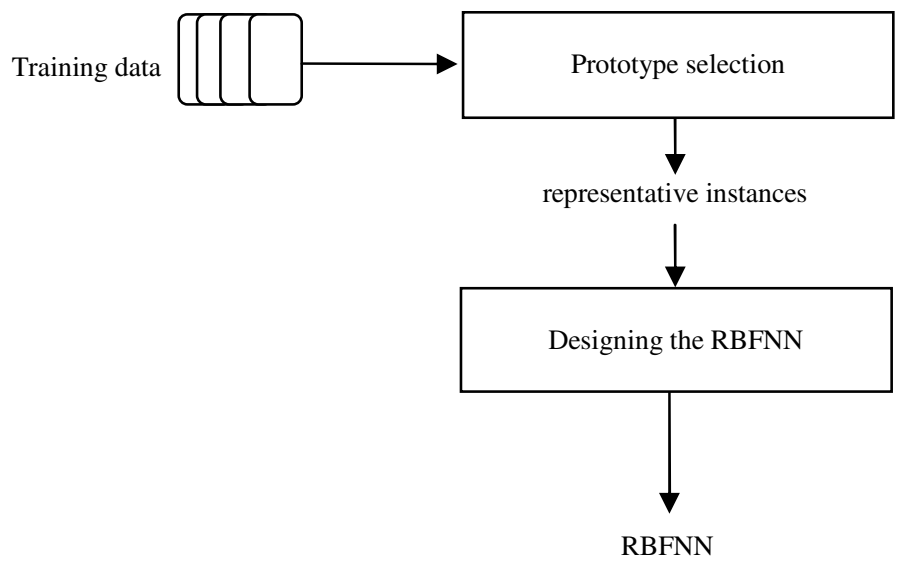

Fig. 2. Proposed Method for designing a RBFNN

The prototype selection methods that we will test for designing the hidden layer of a RBFNN are OSC, CLU and PSR [10,11], these methods are based on clustering and they are the fastest methods for prototype selection [10]. Additionally, we include the DROP3 method which is not as fast as the others but is one of the best prototype selection methods [12].

For our study, we used a RBFNN similar to the one reported in [13], this network uses in the hidden layer a neuron for each instance in the training set. For our method we use a neuron for each instance selected by the prototype selection method. In the 
same way as in [13] we use as vector of means for each neuron the values of its respective instance. The standard deviation is the same for all neurons in the hidden layer. For computing the standard deviation the amount of instances in the training and the number of attributes used for describing them are used in the same way as in [13]. The network is trained using the fast training algorithm steepest descent [13], using negative gradient. This algorithm repeats the training a certain maximum number of times while the accuracy threshold or the maximum number of iterations is not reached.

\section{Experiments}

In this section we present a comparison of the size of the network (number of neurons in the hidden layer), accuracy and runtime needed by our method, for designing and training a RBFNN, against those results obtained by methods based on evolutionary approaches.

Since the authors of original implementations refuse to provide their programs, in order to compare our proposed method against previous approaches for designing RBFNNs, we implement two methods using genetic algorithms and one more using evolution strategies, based on [5] and [7, 14], respectively.

For the genetic approach, GA-RBFNN was implemented based on [5]. In this method, each individual of the population represents a RBFNN configuration (including number of neurons, means and standard deviations). The population is evolved and the best individual is selected. As fitness function, the accuracy of a RBFNN built using the individual (as it was described in section 3) over a validation dataset, is used. The one-point crossover operator is applied using a random crossover-point, and the mutation probability is obtained in a random way at the beginning of the algorithm and it remains the same along all generations. A second variant of GA-RBFNN, called GAS-RBFNN was implemented in a similar way, but the individuals represent a subset of training objects that will be used to build the hidden layer of a RBFNN.

For the evolution strategy, EvRBF [7, 14] was implemented using tournament selection and elitist replacement. The crossover operator consists in interchanging a random number of neurons of the hidden layer, and the mutation operator modifies the means and standard deviation of some neurons of this layer. Additionally, a random number of neurons can be added or eliminated. The fitness function is evaluated as an average, among the training and validation datasets, of the difference between the obtained and the expected outputs of the RBFNN built using an individual.

\subsection{Databases}

For our experiments we used twelve databases taken from the UCI repository [15]. The characteristics of these databases are shown in the Table 1.

\subsection{Experiments}

For our experiments, the prototype selection methods OSC, PSR and CLU were implemented in Matlab [19] and we used the author implementation in C of DROP3 
Table 1. Databases used in the experiments

\begin{tabular}{|l|c|c|c|}
\hline \multicolumn{1}{|c|}{ Databases } & \#Attr & \#Class & \#Inst \\
\hline Glass & 9 & 7 & 214 \\
\hline Pima & 8 & 2 & 768 \\
\hline Yeast & 8 & 10 & 1484 \\
\hline Lymphography & 18 & 4 & 148 \\
\hline Primary-tumor & 17 & 22 & 339 \\
\hline Soybean & 35 & 19 & 307 \\
\hline Waveform & 21 & 3 & 5000 \\
\hline Wines & 13 & 3 & 178 \\
\hline Zoo & 16 & 7 & 101 \\
\hline Sonar & 60 & 2 & 208 \\
\hline Ionosphere & 34 & 2 & 351 \\
\hline Breast-cancer & 9 & 2 & 286 \\
\hline
\end{tabular}

[12]. The RBFNN was implemented in Java 6. The methods based on genetic algorithms were implemented into the package JGAP [20] (in Java). The Evolution stetegy was implemented using the Keel platform [16]. All the methods for designing a RBFNN were executed in a PC having a Mobile AMD Sempron processor at 2 Ghz with $1 \mathrm{~GB}$ in RAM using Windows Vista.

For all the experiments, we used k-fold cross validation with $\mathrm{k}=3$. For each fold, we tried to keep the original proportion of the instances in the classes. Thus, two folds, i.e., the $66 \%$ of the objects in the database were used for training and the remaining $33 \%$ for testing.

It is important to highlight that we included the evaluation of the RBFNN built without applying prototype selection (see column Orig in tables 2, 3 and 4), i.e., using the $66 \%$ of the objects in the database as neurons in the hidden layer of the network, and the remaining $33 \%$ of the objects as testing.

For the proposed method based on prototype selection, the objects selected by OSC, PSR, CLU and DROP3 were used as neurons in the hidden layer. Then the RBFNN was trained, for adjusting the weights, using the $66 \%$ of the objects in the database. And finally the RBFNN was evaluated using the remaining $33 \%$ of objects.

For the evolutionary methods, the RBFNN was designed and trained using the $66 \%$ of the objects in the database. And finally the RBFNN was evaluated using the remaining $33 \%$ of objects.

\subsection{Results}

The number of neurons in the hidden layer of the RBFNNs built by each method is shown in table 2 . In the penultimate row (average 1) of this table we show the average size of the designed RBFNNs excluding the two largest datasets (Yeast and Waveform) because, after 50 hours, the evolutionary methods could not build a RBFNN for these datasets. In the last row (average 2) of table 2, we show the average size of the RBFNNs designed by the methods that could design RNFNNs for all datasets. We can see that the smallest networks were designed applying the Evolution Strategies based method (ES in table 2) followed by the networks designed applying the prototype selection method DROP3 (Drop3 in table 2). However, the accuracies obtained by the networks designed by the Evolution Strategies based method were the 
Table 2. Number of neurons in the hidden layer

\begin{tabular}{|l|c|c|c|c|c|c|c|c|}
\hline \multicolumn{1}{|c|}{ Databases } & Orig & OSC & PSR & Drop3 & CLU & ES & GA & GAS \\
\hline Glass & 142 & 70 & 82 & 31 & 43 & 7 & 46 & 65 \\
\hline Pima & 512 & 169 & 263 & 72 & 154 & 32 & 331 & 106 \\
\hline Yeast & 989 & 421 & 637 & 169 & 297 & 61 & n. a. & n. a. \\
\hline Lymphography & 98 & 43 & 55 & 28 & 29 & 4 & 89 & 33 \\
\hline Primary-tumor & 226 & 121 & 147 & 31 & 68 & 3 & 121 & 144 \\
\hline Soybean & 204 & 126 & 165 & 50 & 61 & 8 & 137 & 184 \\
\hline Waveform & 3333 & 650 & 1803 & 494 & 1000 & n. a. & n. a. & n. a. \\
\hline Wines & 118 & 44 & 70 & 21 & 35 & 2 & 95 & 61 \\
\hline Zoo & 67 & 36 & 43 & 15 & 20 & 4 & 56 & 34 \\
\hline Sonar & 139 & 44 & 70 & 34 & 41 & 9 & 65 & 116 \\
\hline Ionosphere & 234 & 101 & 115 & 17 & 70 & 7 & 154 & 209 \\
\hline Breast-cancer & 191 & 71 & 98 & 26 & 57 & 9 & 147 & 113 \\
\hline Average 1 & $\mathbf{5 2 1 . 1}$ & $\mathbf{1 5 8}$ & $\mathbf{2 9 5 . 7}$ & $\mathbf{8 2 . 3}$ & $\mathbf{1 5 6 . 3}$ & $\mathbf{n . ~ a . ~}$ & $\mathbf{n . ~ a . ~}$ & n. a. \\
\hline Average 2 & $\mathbf{1 9 3 . 1}$ & $\mathbf{8 2 . 5}$ & $\mathbf{1 1 0 . 8}$ & $\mathbf{3 2 . 5}$ & $\mathbf{5 7 . 8}$ & $\mathbf{8 . 5}$ & $\mathbf{1 2 4 . 1}$ & $\mathbf{1 0 6 . 5}$ \\
\hline
\end{tabular}

Table 3. Accuracy

\begin{tabular}{|l|c|c|c|c|c|c|c|c|}
\hline \multicolumn{1}{|c|}{ Databases } & Orig & OSC & PSR & Drop3 & CLU & ES & GA & GAS \\
\hline Glass & 35.52 & 35.52 & 35.51 & 35.52 & 35.52 & 32.75 & 52.80 & 36.90 \\
\hline Pima & 72.66 & 73.17 & 72.65 & 71.62 & 71.62 & 65.75 & 69.27 & 65.90 \\
\hline Yeast & 59.23 & 58.89 & 58.56 & 58.96 & 59.02 & 29.84 & n. a. & n. a. \\
\hline Lymphography & 78.94 & 78.92 & 78.25 & 80.28 & 78.27 & 46.89 & 76.30 & 76.92 \\
\hline Primary-tumor & 44.23 & 43.35 & 38.05 & 27.93 & 21.8 & 20.59 & 32.13 & 42.76 \\
\hline Soybean & 87.28 & 87.29 & 71.66 & 67.08 & 71.65 & 11.16 & 77.48 & 85.65 \\
\hline Waveform & 86.76 & 86.60 & 86.80 & 86.70 & 86.86 & n. a. & n. a. & n. a. \\
\hline Wines & 66.86 & 66.86 & 67.43 & 66.86 & 66.85 & 63.34 & 70.24 & 66.86 \\
\hline Zoo & 84.10 & 86.06 & 84.07 & 83.09 & 88.08 & 61.11 & 94.09 & 85.09 \\
\hline Sonar & 90.39 & 86.05 & 80.29 & 84.13 & 84.13 & 68.27 & 77.43 & 87.99 \\
\hline Ionosphere & 87.46 & 82.62 & 82.62 & 88.88 & 86.61 & 66.66 & 80.91 & 88.03 \\
\hline Breast-cancer & 72.36 & 73.06 & 72.36 & 74.11 & 73.76 & 68.20 & 73.43 & 72.71 \\
\hline Average 1 & $\mathbf{7 2 . 1 5}$ & $\mathbf{7 1 . 5 3}$ & $\mathbf{6 9 . 0 2}$ & $\mathbf{6 8 . 7 6}$ & $\mathbf{6 8 . 6 8}$ & n. a. & n. a. & n. a. \\
\hline Average 2 & $\mathbf{7 1 . 9 8}$ & $\mathbf{7 1 . 2 9}$ & $\mathbf{6 8 . 2 9}$ & $\mathbf{6 7 . 9 5}$ & $\mathbf{6 7 . 8 3}$ & $\mathbf{5 0 . 4 7}$ & $\mathbf{7 0 . 4 1}$ & $\mathbf{7 0 . 8 8}$ \\
\hline
\end{tabular}

worst. The accuracies obtained by the networks designed applying the prototype selection method DROP3 were better but they were not the best.

Table 3 shows the accuracy of the RBFNN built by each method for each dataset. In the penultimate row (average 1) of this table we show the average accuracy of the designed RBFNNs excluding the two largest datasets (Yeast and Waveform) because, after 50 hours, the evolutionary methods could not build a RBFNN for these datasets. In the last row (average 2) of table 3, we show the average accuracy of the RBFNNs designed by the methods that could design RNFNNs for all datasets. Among the method for designing a RNFNN, we can see in table 3 that the best results in accuracy were obtained when the network was designed without applying prototype selection. The second best results in accuracy were obtained by our proposed method using the prototype selection method OSC. The networks designed by the genetic algorithm based methods obtained accuracies close to the obtained by our method, however genetic algorithm based methods require a lot of time for designing the network. Besides, the time needed by the method that does not apply prototype selection is clearly longer than the time needed by our proposed method. 
Table 4 shows the runtimes for designing the RBFNN by each method for each dataset. In the last row of table 4, we show the total runtime needed for each method for designing the RBFNNs for all datasets. In table 4, the columns ES, GA and GAS have some cells where appear $>50$, it means that we stop the method when it reached 50 hours. In the columns ES, GA and GAS instead of the total time appears $>58 \mathrm{~h}$, $>117 \mathrm{~h}$ and $>112 \mathrm{~h}$ respectively since for some databases these methods were stopped when they reached 50 hours, thus for computing the total we used in those databases 50 hours. The method that needed the least amount of time was our method using the prototype selection method DROP3. The second best method was our method using the prototype selection method OSC. However, it is important to remark that, among these methods the method using OSC is the one that obtains the best accuracies.

Taking into account all the above remarks, we can conclude that our method using OSC is the best option for designing a RBFNN since this method designs networks with the best accuracies, which have a size (number of neurons) lesser than the size of the networks designed by genetic algorithm based methods (the most used methods for designing RBFNNs). Additionally, our method requires much shorter time for designing a RBFNN than genetic algorithm based methods.

Table 4. Runtime

\begin{tabular}{|l|c|c|c|c|c|c|c|c|}
\hline \multicolumn{1}{|c|}{ Databases } & Orig & OSC & PSR & Drop3 & CLU & ES & GA & GAS \\
\hline Glass & $00: 01: 51$ & $00: 01: 01$ & $00: 01: 00$ & $00: 00: 26$ & $00: 00: 36$ & $00: 08: 00$ & $00: 18: 56$ & $00: 24: 40$ \\
\hline Pima & $00: 09: 43$ & $00: 03: 20$ & $00: 04: 37$ & $00: 01: 19$ & $00: 02: 45$ & $01: 10: 02$ & $01: 48: 05$ & $01: 45: 55$ \\
\hline Yeast & $08: 20: 36$ & $3: 01: 18$ & $04: 34: 18$ & $00: 59: 53$ & $01: 50: 25$ & $05: 24: 02$ & $>50 \mathrm{~h}$ & $>50 \mathrm{~h}$ \\
\hline Lymphography & $00: 00: 25$ & $00: 00: 15$ & $00: 00: 12$ & $00: 00: 10$ & $00: 00: 12$ & $00: 05: 01$ & $00: 08: 41$ & $00: 07: 05$ \\
\hline Primary-tumor & $00: 22: 30$ & $00: 19: 10$ & $00: 14: 02$ & $00: 03: 18$ & $00: 06: 53$ & $00: 07: 00$ & $04: 40: 51$ & $03: 40: 50$ \\
\hline Soybean & $00: 14: 05$ & $00: 09: 09$ & $00: 11: 40$ & $00: 03: 24$ & $00: 04: 13$ & $00: 17: 00$ & $02: 55: 11$ & $04: 23: 54$ \\
\hline Waveform & $41: 29: 44$ & $05: 28: 20$ & $18: 02: 35$ & $03: 47: 23$ & $08: 42: 28$ & $>50 \mathrm{~h}$ & $>50 \mathrm{~h}$ & $>50 \mathrm{~h}$ \\
\hline Wines & $00: 00: 28$ & $00: 00: 14$ & $00: 00: 18$ & $00: 00: 09$ & $00: 00: 12$ & $00: 04: 00$ & $00: 12: 20$ & $00: 12: 10$ \\
\hline Zoo & $00: 00: 21$ & $00: 00: 11$ & $00: 00: 15$ & $00: 00: 08$ & $00: 00: 10$ & $00: 03: 01$ & $00: 09: 30$ & $00: 9: 51$ \\
\hline Sonar & $00: 00: 26$ & $00: 00: 11$ & $00: 00: 15$ & $00: 00: 10$ & $00: 00: 12$ & $00: 13: 03$ & $06: 45: 06$ & $00: 30: 28$ \\
\hline Ionosphere & $00: 01: 26$ & $00: 00: 18$ & $00: 00: 44$ & $00: 00: 10$ & $00: 00: 29$ & $00: 27: 00$ & $00: 26: 29$ & $00: 52: 11$ \\
\hline Breast-cancer & $00: 00: 53$ & $00: 00: 21$ & $00: 00: 29$ & $00: 00: 10$ & $00: 00: 18$ & $00: 09: 01$ & $00: 18: 53$ & $00: 21: 48$ \\
\hline Total time & $\mathbf{5 0 : 4 2 : 2 8}$ & $\mathbf{9 : 0 3 : 4 8}$ & $\mathbf{2 3 : 1 0 : 2 5}$ & $\mathbf{4 : 5 6 : 4 0}$ & $\mathbf{1 0 : 4 8 : 2 9}$ & $>\mathbf{5 8 ~ h}$ & $>\mathbf{1 1 7} \mathbf{~ h}$ & $>\mathbf{1 1 2} \mathbf{~ h}$ \\
\hline
\end{tabular}

\section{Conclusions}

In this paper, we propose a method for the automatic design of a RBFNN based on prototype selection.

Based on the experimental results we can conclude that the proposed method, using OSC as prototype selection method, is the best option for designing a RBFNN since this method designs networks with the best accuracies, which have a size (number of neurons) smaller than the size of the networks designed by genetic algorithm based methods (the most used methods for designing RBFNNs). Additionally, our method requires much shorter time for designing a RBFNN than genetic algorithm based methods.

As future work, we will include in our method some strategies to simultaneously adjust the parameters of a RBFNN. 


\section{References}

1. Yao, X.: Evolving artificial neural networks. Proceedings of the IEEE 87(9), 1423-1447 (1999)

2. Tian, J., Li, M., Chen, F.: A Cooperative Coevolution Algorithm of RBFNN for Classification. In: Zhou, Z.-H., Li, H., Yang, Q. (eds.) PAKDD 2007. LNCS (LNAI), vol. 4426, pp. 809-816. Springer, Heidelberg (2007)

3. Qin, Z., Chen, J., Liu, Y., Lu, J.: Evolving RBF Neural Networks for Pattern Classification. In: Hao, Y., Liu, J., Wang, Y.-P., Cheung, Y.-m., Yin, H., Jiao, L., Ma, J., Jiao, Y.-C. (eds.) CIS 2005. LNCS (LNAI), vol. 3801, pp. 957-964. Springer, Heidelberg (2005)

4. Chen, Y., Yang, B., Zhou, J.: Automatic Design of Hierarchical RBF Networks for System Identification. In: Yang, Q., Webb, G. (eds.) PRICAI 2006. LNCS (LNAI), vol. 4099, pp. 1191-1195. Springer, Heidelberg (2006)

5. Tian, J., Li, M., Chen, F.: Improving multiclass pattern recognition with a co-evolutionary RBFNN. Pattern Recognition Letters 29(4), 392-406 (2008)

6. Tian, J., Li, M., Chen, F.: A hybrid classification algorithm based on co-evolutionary EBFNN and domain covering method. Neural Computing \& Applications 18(3), 293-308 (2009)

7. Parras-Gutierrez, E., Rivas, V.M., Del Jesus, M.J.: Automatic Neural Net Design by Means of a Symbiotic Co-evolutionary Algorithm. In: Corchado, E., Abraham, A., Pedrycz, W. (eds.) HAIS 2008. LNCS (LNAI), vol. 5271, pp. 140-147. Springer, Heidelberg (2008)

8. Mu, S., Tian, S., Yin, C.: A Novel Radial Basis Function Neural Network Classifier with Centers Set By Cooperative Clustering. International Journal of Fuzzy Systems 9(4), 205$211(2007)$

9. Pedrycz, W., Park, H.S., Oh, S.K.: A granular-oriented development of functional radial basis function neural networks. Neurocomputing 72(1-3), 420-435 (2008)

10. Olvera-Lopez, A., Carrasco-Ochoa, J.A., Martinez-Trinidad, J.F.: Object Selection Based on Clustering and Border Objects. Computer Recognition Systems 2, Advances in Soft Computing 45, 27-34 (2007)

11. Lumini, A., Nanni, L.: A clustering method for automatic biometric template selection. Pattern Recognition 39(3), 495-497 (2006)

12. Wilson, D.R., Martinez, T.R.: Reduction Techniques for Instance-Based Learning Algorithms. Machine Learning 38(3), 257-286 (2000)

13. Looney, C.G.: Pattern Recognition Using Neural Networks. In: Theory and Algorithms for Engineers and Scientists. Oxford University Press, Oxford (1997)

14. Rivas, V.M., Merelo, J.J., Castillo, P.A., Arenas, M.G., Castellano, J.G.: Evolving RBF neural networks for time-series forecasting with EvRBF. Information Sciences 165(3-4), 207-220 (2003)

15. Blake, C., Keogh, E., Merz, C.J.: UCI repository of machine learning databases, Department of Information and Computer Science, University of California, Irvine, CA (1998), http: / / www.ics.uci.edu/ mlearn/MLRepository.html

16. Alcalá-Fdez, J., Sánchez, L., García, S., del Jesus, M.J., Ventura, S., Garrell, J.M., Otero, J., Romero, C., Bacardit, J., Rivas, V.M., Fernández, J.C., Herrera, F.: KEEL: A Software Tool to Assess Evolutionary Algorithms to Data Mining Problems. Soft Computing 13(3), 307-318 (2009) 
17. Haykin, S.: Neural Networks: a comprehensive foundation, 2nd edn. Prentice Hall, Englewood Cliffs (2005)

18. Konar, A.: Computational Intelligence: principles, techniques, and applications. Springer, Heidelberg (2005)

19. The MathWorks Inc., Natick (1994-2008), http : / / www . mathworks . com

20. Meffert, Klaus, et al.: JGAP - Java Genetic Algorithms and Genetic Programming Package, http://jgap.sf.net 